Pacific Journal of Mathematics

MOMENT SEQUENCES IN HILBERT SPACE 


\section{MOMENT SEQUENCES IN HILBERT SPACE}

\section{GoRDON G. JoHNSON}

Suppose $f$ is a real valued function of bounded variation on $[0,1]$. Then for each nonnegative integer $n$, the Stieltjes integral $\int_{0}^{1} j^{n} d f$ exists, where for each number $x, j(x)=x$. A necessary and sufficient condition is given for $f$ in order that the moment sequence for $f,\left\{C_{n}\right\}_{n=0}^{\infty}$, is square summable. A second result establishes that the set of all such square summable moment sequences is dense in $l^{2}$.

LEMMA 1. If $p$ is a number, $1 / 2<p<1$, and for each nonnegative integer $n, a_{n}=1-(n+1)^{-p}$ then

$$
\begin{aligned}
& \text { 1. } \lim _{n \rightarrow \infty} a_{n}^{n}=0 \text {, } \\
& \text { 2. } \sum_{n=0}^{\infty} a_{n}^{2 n} \text { exists }
\end{aligned}
$$

and

$$
\text { 3. } \sum_{n=0}^{\infty}\left(1-a_{n}\right)^{2} \text { exists . }
$$

Proof. To establish 1,

$$
\begin{aligned}
\lim _{n \rightarrow \infty} a_{n}^{n} & =\lim _{n \rightarrow \infty}\left(1-n^{-p}\right)^{n} \\
& =\exp \left[\lim _{n \rightarrow \infty} n \ln \left[1-n^{-p}\right]\right] .
\end{aligned}
$$

Since $1 / 2<p<1$,

$$
\lim _{n \rightarrow \infty} n \ln \left[1-n^{-p}\right]=-p \lim _{n \rightarrow \infty} n /\left[n^{p}-1\right]=-\infty
$$

and hence the result.

To establish 2, it will be sufficient to show that for sufficiently large $n$

$$
a_{n}^{n} \leqq(1+n)^{-p}
$$

i.e., that $\left[1-n^{-p}\right]^{n-1} \leqq n^{-p}$.

Let $n^{p}=k$ and $g=p^{-1}-1$ (note that $g>0$ ); we have then to show that

$$
\left[\left[1-k^{-1}\right]^{k}\right]^{k^{g}} \leqq k^{-1}-k^{-2}
$$

Recall that 


$$
\left[1-k^{-1}\right]^{k} \leqq e^{-1}
$$

and hence that

$$
\left[\left[1-k^{-1}\right]^{k}\right]^{k^{g}} \leqq e^{-k^{g}}
$$

Now if $k$ is large we have

$$
e^{-k^{g}} \leqq k^{-1}-k^{-2}
$$

and the result is established.

The third part follows immediately from the definition of $a_{n}$.

THEOREM 1. If $f$ is a real valued function of bounded variation on $[0,1]$ and, for each nonnegative integer $n, \int_{0}^{1} j^{n} d f=C_{n}$ exists, then

$$
\sum_{n=0}^{\infty} C_{n}^{2}<\infty
$$

if and only if

$$
\sum_{n=1}^{\infty}\left[f(1)-\int_{a_{n}}^{1} f d j^{n}\left(1-a_{n}^{n}\right)\right]^{2}<\infty
$$

where the sequence $\left\{a_{n}\right\}_{n=0}^{\infty}$ is as given in Lemma 1.

Proof. Let us first establish the necessity of the condition. Suppose $\sum_{n=0}^{\infty} C_{n}^{2}<\infty$.

If $n$ is a positive integer

$$
\begin{aligned}
C_{n} & =\int_{0}^{1} j^{n} d f \\
& =\int_{0}^{a_{n}} j^{n} d f+\int_{a_{n}}^{1} j^{n} d f \\
& =a_{n}^{n} f\left(a_{n}\right)-\int_{0}^{a_{n}} f d j^{n}+\int_{a_{n}}^{1} j^{n} d f .
\end{aligned}
$$

Let $\gamma_{n}=\int_{0}^{a_{n}} f d j^{n} / a_{n}^{n}$, then

$$
C_{n}=a_{n}^{n}\left[f\left(a_{n}\right)-\gamma_{n}\right]+f(1)-f\left(a_{n}\right) a_{n}^{n}-\int_{a_{n}}^{1} f d j^{n}
$$

Let $\delta_{n}=\int_{a_{n}}^{1} f d j^{n} /\left(1-a_{n}^{n}\right)$, then

$$
\begin{aligned}
& C_{n}=a_{n}^{n}\left[f\left(a_{n}\right)-\gamma_{n}\right]+f(1)-f\left(a_{n}\right) a_{n}^{n}-\left(1-a_{n}^{n}\right) \delta_{n} \\
& C_{n}=a_{n}^{n}\left[\delta_{n}-\gamma_{n}\right]+\left[f(1)-\delta_{n}\right]
\end{aligned}
$$

and 


$$
C_{n}^{2}=\left(a_{n}^{n}\left[\delta_{n}-\gamma_{n}\right]+\left[f(1)-\delta_{n}\right]\right)^{2} .
$$

Since the sequence $\left\{\left[\delta_{n}-\gamma_{n}\right]\right\}_{n=1}^{\infty}$ is bounded it follows from Lemma 1 that

$$
\sum_{n=1}^{\infty} \alpha_{n}^{2 n}\left[\delta_{n}-\gamma_{n}\right]^{2}<\infty
$$

Hence, since $\sum_{n=0}^{\infty} C_{n}^{2}<\infty$, we have that

$$
\sum_{n=1}^{\infty}\left[f(1)-\delta_{n}\right]^{2}<\infty
$$

i.e.,

$$
\sum_{n=1}^{\infty}\left|f(1)-\int_{a_{n}}^{1} f d j^{n} /\left(1-a_{n}^{n}\right)\right|^{2}<\infty
$$

and therefore the condition is necessary.

Now let us establish the sufficiency, i.e., suppose that

$$
\sum_{n=1}^{\infty}\left|f(1)-\int_{a_{n}}^{1} f d j^{n} /\left(1-a_{n}^{n}\right)\right|^{2}
$$

exists.

Now $C_{n}=\left[f(1)-\int_{a_{n}}^{1} f d j^{n}\right]-\int_{0}^{a_{n}} f d j^{n}$ for $n=0,1,2, \cdots$.

As befor

$$
\sum_{n=1}^{\infty}\left(\int_{0}^{a_{n}} f d j^{n}\right)^{2}
$$

exists and hence we have only to consider

$$
\begin{aligned}
& \sum_{n=1}^{\infty}\left(f(1)-\int_{a_{n}}^{1} f d j^{n}\right)^{2} \\
= & \sum_{n=1}^{\infty}\left(\left[f(1)-\int_{a_{n}}^{1} f d j^{n}\right] /\left[1-a_{n}^{n}\right]\right)^{2}\left(1-a_{n}^{n}\right)^{2} \\
\leqq & \sum_{n=1}^{\infty}\left(\left[f(1)-\int_{a_{n}}^{1} f d j^{n}\right] /\left[1-a_{n}^{n}\right]\right)^{2} \\
= & \sum_{n=1}^{\infty}\left(f(1)-\int_{a_{n}}^{1} f d j^{n} /\left[1-a_{n}^{n}\right]+f(1) a_{n}^{n} /\left[1-a_{n}^{n}\right]\right)^{2} .
\end{aligned}
$$

Recall the assumption that

$$
\sum_{n=1}^{\infty}\left(f(1)-\int_{a_{n}}^{1} f d j^{n} /\left[1-a_{n}^{n}\right]\right)^{2}
$$

exists and hence we need only consider 


$$
\begin{aligned}
& \sum_{n=1}^{\infty}\left(f(1) a_{n}^{n} /\left[1-a_{n}^{n}\right]\right)^{2} \\
= & \sum_{n=1}^{\infty}(f(1))^{2} \alpha_{n}^{2 n} /\left[1-a_{n}^{n}\right]^{2}
\end{aligned}
$$

which also exists. Hence it follows that $\sum_{n=0}^{\infty} C_{n}^{2}$ exists.

As an immediate consequence of this result we have the following results, which are stated here without proof.

Proposition 1. If there is a $\delta, 0<\delta<1$, such that $f(1)-f(x) \leqq$ $1-x$ if $\delta \leqq x \leqq 1$ then $\sum_{n=0}^{\infty} C_{n}^{2}<\infty$.

Proposition 2. If there is a $\delta, 0<\delta<1$, such that $f$ has a continuous derivative on $[\delta, 1]$ then $\sum_{n=0}^{\infty} C_{n}^{2}<\infty$.

Proposition 3. If there is a number $\delta, 0<\delta<1$, a number $\alpha>1 / 2$ and $a$ number $B>0$ such that

$$
|f(1)-f(x)| \leqq B|1-x|^{\alpha} \text { for } x \text { in }[\delta, 1]
$$

then $\sum_{n=0}^{\infty} C_{n}^{2}<\infty$.

Consider the following example. Let $f=1-(1-j)^{1 / 2}$ on $[0,1]$, then $C_{n}=\int_{0}^{1} j^{n} d f=2 n \int_{0}^{1} j^{2}\left(1-j^{2}\right)^{n-1}$ if $n \geqq 1$, and hence $C_{n+1}=2(n+$ 1) $\int_{0}^{1} j^{2}\left(1-j^{2}\right)^{n}$. It then follows that $(2 n+3) C_{n+1}=(2 n+2) C_{n}$ and this yields the following for $n=1,2, \cdots, C_{n+1}=C_{1} \prod_{t=0}^{n-1}[(2 t+4)((2 t+5)]$. By the use of Stirlings formula we have that $C_{n+1}^{2} \geqq 6 \sqrt{\pi}(n+3 / 2)^{-1 / 2}$ and hence $\sum_{n=0}^{\infty} C_{n}^{2}$ does not exist.

The following lemma is stated without proof.

Lemma 2. If $t$ is a positive integer and $n$ is a nonnegative integer less than $t$, then

$$
\sum_{m=0}^{t}\left(\begin{array}{l}
t \\
m
\end{array}\right) m^{t}(-1)^{m}=(-1)^{t} t !
$$

and

$$
\sum_{m=0}^{t}\left(\begin{array}{l}
t \\
m
\end{array}\right) m^{n}(-1)^{m}=0
$$

Definition 1. Suppose $\left\{C_{m}\right\}_{m=0}^{\infty}$ is a real number sequence and $n$ is a positive integer. Let $\varphi_{n}(0)=0, \varphi_{n}(1)=C_{0}$ and if $x$ is in $(0,1) \cap$ $[k / n,(k+1) / n)$ where $k=0,1,2, \cdots, n-1$ let 


$$
\varphi_{n}(x)=\sum_{t=0}^{k}\left(\begin{array}{l}
n \\
t
\end{array}\right) \sum_{i=0}^{n-t}\left(\begin{array}{c}
n-t \\
i
\end{array}\right)(-1)^{i} C_{i+t}
$$

THEOREM 2. The set of all square summable moment sequences is dense in $l^{2}$.

Proof. Let, for each nonnegative integer $t, \varepsilon_{t}=\left\{\delta_{i t}\right\}_{i=0}^{\infty}$ where $\delta_{i j}$ is the Kronecker $\delta$. Associated with each such sequence $\varepsilon_{t}$, there is a function sequence $\left\{\varphi_{k, t}\right\}_{k=1}^{\infty}$ as given in Definition 1. For each nonnegative integer $t$ and each positive integer $k$ there is a number sequence $C_{k, t}=\left\{C_{n, k, t}\right\}_{n=0}^{\infty}$ associated, where $C_{n, k, t}=\int_{0}^{1} j^{n} d \varphi_{k, t}$.

A straight forward computation yields

$$
\begin{aligned}
C_{n, k, t} & =\sum_{m=0}^{t}(-1)^{t-m}(m / k)^{n}\left(\begin{array}{c}
k \\
m
\end{array}\right)\left(\begin{array}{c}
k-m \\
t-m
\end{array}\right) \\
& =(-1)^{t}\left(\begin{array}{l}
k \\
t
\end{array}\right) \sum_{m=0}^{t}\left(\begin{array}{c}
t \\
m
\end{array}\right)(-1)^{m}(m / k)^{n}
\end{aligned}
$$

and therefore

$$
\sum_{n=0}^{\infty} C_{n, k, t}^{2}=\sum_{n=0}^{\infty}\left(\begin{array}{c}
k \\
t
\end{array}\right)^{2}\left[\sum_{m=0}^{t}\left(\begin{array}{c}
t \\
m
\end{array}\right)(-1)^{m}(m / k)^{n}\right]^{2}
$$

This, using Lemma 2, becomes

$$
\begin{aligned}
& \sum_{n=t}^{\infty}\left(\begin{array}{c}
k \\
t
\end{array}\right)^{2}\left[\sum_{m=0}^{t}\left(\begin{array}{c}
t \\
m
\end{array}\right)(-1)^{m}(m / k)^{n}\right]^{2} \\
= & \left.\sum_{n=0}^{\infty}\left(\begin{array}{c}
k \\
t
\end{array}\right)^{2}\left[\begin{array}{c}
t \\
m=0 \\
m
\end{array}\right)\left(\begin{array}{c}
t \\
m
\end{array}\right)(-1)^{m}(m / k)^{n}(m / k)^{t}\right]^{2} \\
= & \sum_{n=0}^{\infty}\left(\begin{array}{c}
k \\
t
\end{array}\right)^{2} k^{-2 t}\left[\sum_{m=0}^{t}\left(\begin{array}{c}
t \\
m
\end{array}\right)^{2}\left(m^{2} / k^{2}\right)^{n} m^{2 t}\right. \\
& \left.+2 \sum_{m=0}^{t-1}\left(\begin{array}{c}
t \\
m
\end{array}\right) m^{t}(m / k)^{n}(-1)^{m} \sum_{i=m+1}^{t}\left(\begin{array}{l}
t \\
i
\end{array}\right) i^{t}(i / k)^{n}(-1)^{i}\right] \\
= & \left(\begin{array}{c}
k \\
t
\end{array}\right)^{2} k^{-2 t}\left[\begin{array}{c}
t \\
m=0
\end{array}\left(\begin{array}{c}
t \\
m
\end{array}\right)^{2} m^{2 t} k^{2} /\left(k^{2}-m^{2}\right)\right. \\
& \left.+2 \sum_{m=0}^{t-1}\left(\begin{array}{c}
t \\
m
\end{array}\right) m^{t}(-1)^{m} \sum_{i=m+1}^{t}\left(\begin{array}{c}
t \\
i
\end{array}\right) i^{t}(-1)^{i} k^{2} /\left(k^{2}-m i\right)\right] \\
= & \sum_{m=0}^{t}\left(\begin{array}{c}
t \\
m
\end{array}\right)^{2} m^{2 t}\left(\begin{array}{c}
k \\
t
\end{array}\right)^{2} k^{-2 t} k^{2} /\left(k^{2}-m^{2}\right) \\
& +2 \sum_{m=0}^{t-1}\left(\begin{array}{c}
t \\
m
\end{array}\right) m^{t}(-1)^{m} \sum_{i=m+1}^{t}\left(\begin{array}{l}
t \\
i
\end{array}\right) i^{t}(-1)^{i}\left(\begin{array}{l}
k \\
t
\end{array}\right)^{2} k^{-2 t} k^{2} /\left(k^{2}-m i\right)
\end{aligned}
$$


if $k>t$.

Note that

$$
\lim _{k \rightarrow \infty} k^{-2 t} k^{2}\left(\begin{array}{l}
k \\
t
\end{array}\right)^{2} /\left(k^{2}-m^{2}\right)=(t !)^{-2}
$$

and that

$$
\lim _{k \rightarrow \infty} k^{-2 t} k^{2}\left(\begin{array}{l}
k \\
t
\end{array}\right)^{2} /\left(k^{2}-m i\right)=(t !)^{-2} .
$$

Then it follows that

$$
\begin{aligned}
& \lim _{k \rightarrow \infty} \sum_{n=0}^{\infty} C_{n, k, t}^{2}=\sum_{m=0}^{t}\left(\begin{array}{c}
t \\
m
\end{array}\right)^{2} m^{2 t}(t !)^{-2} \\
& +2 \sum_{m=0}^{t-1}\left(\begin{array}{c}
t \\
m
\end{array}\right) m^{t}(-1)^{m} \sum_{i=m+1}^{t}\left(\begin{array}{l}
t \\
i
\end{array}\right) i^{t}(-1)^{i}(t !)^{-2} \\
& =(t !)^{-2}\left[\sum_{m=0}^{t}\left(\begin{array}{c}
t \\
m
\end{array}\right) m^{t}(-1)^{m}\right]^{2} \\
& =1 \text {. }
\end{aligned}
$$

Hence, if $t$ is a nonnegative integer

$$
\lim _{k \rightarrow \infty}\left\|C_{k, t}\right\|=1 \cdot\left(\|\cdot\| \text { is } l^{2} \text { norm }\right)
$$

Let us now show that

$$
\lim _{k \rightarrow \infty}\left\|\varepsilon_{t}-\varepsilon_{k, t}\right\|=0 .
$$

Suppose $t$ is a nonnegative integer and $k$ is a positive integer greater than $t$.

$$
\begin{aligned}
\sum_{n=0}^{\infty}\left(\delta_{n, t}\right. & \left.-C_{n, k, t}\right)^{2} \\
& =\sum_{n=t}^{\infty}\left(\delta_{n, t}-C_{n, k, t}\right)^{2} \\
& =\left(\delta_{t, t}-C_{t, k, t}\right)^{2}+\sum_{n=t+1}^{\infty} C_{n \cdot k, t}^{2} \\
& =\left(1-C_{t, k, t}\right)^{2}+\sum_{n=t+1}^{\infty} C_{n, k, t}^{2} .
\end{aligned}
$$

Now

$$
\left(1-C_{t, k, t}\right)^{2}=\left(1-\left(\begin{array}{c}
k \\
t
\end{array}\right) k^{-t} \sum_{m=0}^{t}\left(\begin{array}{c}
t \\
m
\end{array}\right) m^{t}(-1)^{m+t}\right)^{2}
$$




$$
\lim _{k \rightarrow \infty}\left(1-C_{t, k, t}\right)^{2}=\left[1-(t !)^{-1} \sum_{m=0}^{t}\left(\begin{array}{c}
t \\
m
\end{array}\right) m^{t}(-1)^{m+t}\right]^{2}
$$

since

$$
\lim _{k \rightarrow \infty}\left(\begin{array}{l}
k \\
t
\end{array}\right) k^{-t}=(t !)^{-1}
$$

and hence by Lemma 2

$$
\lim _{k \rightarrow \infty}\left(1-C_{t, k, t}\right)^{2}=0
$$

Combining this with the fact that

$$
\sum_{n=0}^{\infty} C_{n, k, t}^{2}=1
$$

yields, $\lim _{k \rightarrow \infty}\left\|\varepsilon_{t}-\varepsilon_{k, t}\right\|=0$ for each nonnegative integer $t$.

Since $\left\{\varepsilon_{t}: t=0,1,2, \cdots\right\}$ is a complete orthonormal set for $l^{2}$ and each point can be approximated by a square summable moment sequence, it follows that the set of all square summable moment sequences is dense in $l^{2}$ and hence the theorem is established.

\section{REFERENCES}

1. R. P. Boas, The Stieltjes moment problem for functions of bounded variation, Bull. Amer. Math. Soc., 45 (1939), 399-404.

2. L. L. Dines, Convex extension and linear inequalities, Bull. Amer. Math. Soc., 42 (1936), 353-365.

3. P. Halmos, Introduction to Hilbert Space, Chelsea Pub. Co., 1957.

4. A. Jakimouski, Some remarks on the moment problem of Hausdorff, J. London Math. Soc., 33 (1958), 1-13.

5. G. G. Johnson, Concerning local variations in the moment problem, J. London Math. Soc., 41 (1966), 667-672.

6. - Concerning local flatness in the moment problem, Portugaliae Math., (3), 28 (1969) 137-149.

7. J. S. MacNerney, Hermitian moment sequences, Trans. Amer. Math. Soc., 103 (1962), $45-81$.

8. H. S. Wall, Continued Fractions, Chelsea Pub. Co., 1957.

9. J. H. Wells, Concerning the Hausdorff inclusion problem, Duke Math. J., 26 (1959), 629-645.

Received January 7, 1972 and in revised form April 24, 1972.

UNIVERSITY OF HOUSTON 



\section{PACIFIC JOURNAL OF MATHEMATICS}

\section{EDITORS}

D. Gilbarg and J. Milgram Stanford University

Stanford, California 94305

R. A. Beaumont

University of Washington

Seattle, Washington 98105
J. DugundJI

Department of Mathematics

University of Southern California

Los Angeles, California 90007

RICHARD ARENS

University of California

Los Angeles, California 90024

ASSOCIATE EDITORS
E. F. BECKENBACH
B. H. NeumanN
F. WOLF
K. YoSHIDA

\section{SUPPORTING INSTITUTIONS}

UNIVERSITY OF BRITISH COLUMBIA

CALIFORNIA INSTITUTE OF TECHNOLOGY

UNIVERSITY OF CALIFORNIA

MONTANA STATE UNIVERSITY

UNIVERSITY OF NEVADA

NEW MEXICO STATE UNIVERSITY

OREGON STATE UNIVERSITY

UNIVERSITY OF OREGON

OSAKA UNIVERSITY
UNIVERSITY OF SOUTHERN CALIFORNIA

STANFORD UNIVERSITY

UNIVERSITY OF TOKYO

UNIVERSITY OF UTAH

WASHINGTON STATE UNIVERSITY

UNIVERSITY OF WASHINGTON

$\stackrel{*}{*} \stackrel{*}{*} \stackrel{*}{*}$ AMERICAN MATHEMATICAL SOCIETY

NAVAL WEAPONS CENTER 


\section{Pacific Journal of Mathematics}

\section{Vol. 46, No. $1 \quad$ November, 1973}

Allan Francis Abrahamse, Uniform integrability of derivatives on $\sigma$-lattices .......................................... 1

Ronald Alter and K. K. Kubota, The diophantine equation $x^{2}+D=p^{n} \ldots \ldots \quad 11$

Grahame Bennett, Some inclusion theorems for sequence spaces .......... 17

William Cutler, On extending isotopies ........................ 31

Robert Jay Daverman, Factored codimension one cells in Euclidean

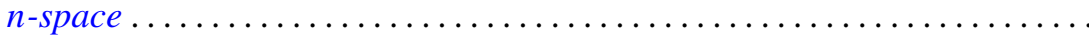

Patrick Barry Eberlein and Barrett O’Neill, Visibility manifolds ............ 45

M. Edelstein, Concerning dentability .......................... 111

Edward Graham Evans, Jr., Krull-Schmidt and cancellation over local

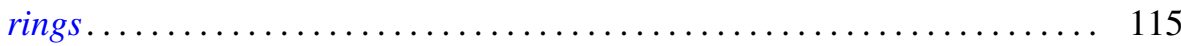

C. D. Feustel, A generalization of Kneser's conjecture ................ 123

Avner Friedman, Uniqueness for the Cauchy problem for degenerate parabolic equations .......................................... 131

David Golber, The cohomological description of a torus action ............ 149

Alain Goullet de Rugy, Un théorème du genre "Andô-Edwards" pour les

Fréchet ordonnés normaux..............................

Louise Hay, The class of recursively enumerable subsets of a recursively enumerable set ........................................

John Paul Helm, Albert Ronald da Silva Meyer and Paul Ruel Young, On orders of translations and enumerations...

Julien O. Hennefeld, A decomposition for $B(X)^{*}$ and unique Hahn-Banach

extensions

Gordon G. Johnson, Moment sequences in Hilbert space .

Thomas Rollin Kramer, A note on countably subparacompact spaces ...

Yves A. Lequain, Differential simplicity and extensions of a derivation ....

Peter Lorimer, A property of the groups Aut $\mathrm{PU}\left(3, q^{2}\right) \ldots$

225

Yasou Matsugu, The Levi problem for a product manifold.

231

John M.F. O'Connell, Real parts of uniform algebras ......

William Lindall Paschke, A factorable Banach algebra without bounded

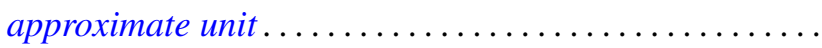

Ronald Joel Rudman, On the fundamental unit of a purely cubic field ....

Tsuan Wu Ting, Torsional rigidities in the elastic-plastic torsion of simply connected cylindrical bars .........................

Philip C. Tonne, Matrix representations for linear transformations on analytic sequences...................................

Jung-Hsien Tsai, On E-compact spaces and generalizations of perfect

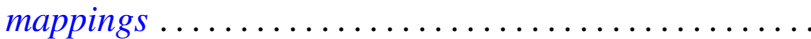

Alfons Van Daele, The upper envelope of invariant functionals majorized by an invariant weight. .. 